\title{
Survivin -31G/C promoter polymorphism and P53 antibodies in breast cancer
}

\author{
Nagwa.S.Ahmed ${ }^{1}$,Madeha M.zakhary ${ }^{2}$,Saad El- Din A. El- \\ Noeman $^{3}$,Shimaa.B.Hemdan ${ }^{4}$ \\ 1-Professor and Head of Biochemistrydepartment,Faculty of Medicine,Sohag University \\ 2-Professor of Medical Biochemistry,Faculty of Medicine,Assiut University \\ 3- Professor of Medical Biochemistry,Faculty of Medicine,Tanta University \\ 4-Porfessor of Biochemistry department,Faculty of Medicine,Sohag University \\ 5-Assistant lecturer of Biochemistry department,Faculty of Medicine,Sohag University
}

Breast cancer has attracted increasing attention by the international community and has evoked tremendous interest paritcularly in the medical and academic fields (Siegel et al.,2015).It is the most common cancer and also the leading cause of cancer mortality in women worldwide .It is a heterogeneous disease with different histological types. Ductal breast cancer constitutes the vast majority of the breast cancers. Breast cancer incidence in developed countries is higher, while relative mortality is greatest in less developed countries. Education of women is suggested in all countries for early detection and treatment. Early detection decreases breast cancer mortality (Monticciolo et al.,2018).

An increasing number of studies have been performed to explore potential biomarkers which may be involved in the initiation and progression of breast cancer. Estrogen receptor (ER), progesterone receptor (PR), c-erbB-2, p53 and Ki-67 have been investigated in the conventional histopathologicalsetting(Ding.,2017 and Parsa et al.,2016).

\section{SURVIVIN STRUCTURE AND FUNCTIONS}

Survivin is an antiapoptotic protein belonging to the inhibitor of apoptosis protein family. It is a bifunctional protein that regulates cell division and suppresses apoptosis. Survivin is highly expressed in various human malignancies, but its expression is very low or below the level of detection in normal adult tissues (Yazdani et al., 2012). The gene encoding human survivin was cloned by Ambrosini et al in 1997. Survivin spans $14.7 \mathrm{~kb}$ at the telomeric end of chromosome 17 and encodes the $16.5 \mathrm{kD}$ wild-type survivin protein of 142 amino acids in length (Ambrosini et al.,1997). DNA polymorphisms with more than one variant (allele) having a frequency greater than 1 per cent in a human population have been estimated to occur on the average at one in every 1000 base pairs throughout the human genome (Sherry et al.,1999). The incidences of polymorphism in genomic DNA, their susceptibility to genetic alterations, and the risk of tumour progression in patients with cancer can vary substantially between different racial groups ( Perez et al .,2006 and Katkoori et al.,2009). Although most polymorphisms are functionally neutral, some affect regulation of gene expression or the function of the coded protein (Costa et al. ,2008).

The survivin gene codifies a multifunctional protein involved in the regulation of the cell cycle and inhibition of the apoptotic pathway, and a polymorphism located in its promoter region is associated with gene regulation. Most of the polymorphic studies are confined in promoter region among which the single nucleotide polymorphism (SNP) at $-31 \mathrm{G} / \mathrm{C}$ is most studied in all cancers in comparison to other 
polymorphic sites (Jaiswal et al.,2012 and Theodoropoulos et al.,2010). The aim of our work was to investigate $-31 \mathrm{G} / \mathrm{C}$ single nucleotide polymorphism of survivin promoter in breast cancer patients.

In malignant cells, all these isoforms are expressed at a very high rate as compared with normal tissues. Survivin has a dual function, playing both a role in cell death regulation and mitotic progression. Survivin can be co-immunoprecipitated with caspases-3, -7, and -9 and it suppresses apoptosis induced by overexpression of these caspases, implying that survivin also is a caspase inhibitor. It also inhibits cell death by interfering with caspase- 9 processing, the main inhibitor in intrinsic pathway of apoptosis (Altieri.,2006).

\section{Survivin and apoptosis}

Activation of cell death pathways can be initiated through different mechanisms, including through ligand binding (FasL, TNF) to a death receptor on the cell surface (extrinsic pathway) or via direct mitochondrial signaling (intrinsic pathway). The mitochondrial pathway is initiated by activation of the Bax/Bcl-2 pathway leading to the release of apoptotic factors such as cytochrome c (cyt c) and apoptosis-inducing factor (AIF) from the mitochondrial intermembrane space into the cytoplasm. The release of cytochrome c from mitochondria results in caspase- 3 activation through formation of the cytochrome c/Apaf-1/caspase- 9 apoptosome complex. Caspase-3 cleaves a number of substrates including cytoskeletal proteins and DNA. Caspaseactivated DNase (CAD) and inhibitor of CAD (ICAD) initiate cleavage and fragmentation of DNA. The IAPs inhibit cell death by physically interacting with caspases. Survivin has been shown to inhibit apoptosis through caspase-dependent and independent pathways. Consistent with the lack of a structural caspase activation and recruitment domain (CARD) motif, survivin does not directly bind to and inhibit caspases1 ( $\mathbf{L i}$,et al.,2008). Instead, it interacts with several adaptor or cofactor molecules, one example being X-linked IAP (XIAP). By interacting with XIAP, survivin enhances XIAP stability (Sensintaffaret al.,2010).

Survivin is a target of p53 for its action and downregulation, and p53 may induce apoptosis by antagonizing the anti-apoptotic activity of survivin.However, the negative regulation of survivin by p53 is poorly understood. Survivin promoter has a p53 binding element. It may be possible that p53 directly binds survivin promoter alone or in combination with other protein(s) to repress survivin. E2F (a transcriptional activator) may also bind survivin promoter (Jiang et al.,2004).

Since p53 has affinity with E2F, it is possible that both form a (p53-E2F) complex that represses survivin gene expression (. It also interacts with transcriptional repressor $(\sin 3)$ and histone deacetylases (HD AC) that together can form a p53-sin3HD AC complex and binds survivin promoter to repress it. $p 53$ represses survivin through a cascade, which involves protein $\mathrm{p} 21$ complex $\mathrm{p} 21 /$ cip1, which is a $\mathrm{p} 53$ induced gene that inhibits cyclindependent kinase $2(\mathrm{cdk} 2)$ to prevent phosphorylation of retinoblastoma (RB) proteins( Hoffman et al.,2015).

This results in the accumulation of hypophosphorylatedpRB. This protein binds E2F family transcription factor and forms a pRB-E2F complex, which may repress survivin gene expression (Hoffman et al.,2015).Survivin expression and its role in different cancer regulatory mechanisms of survivin expression are yet not fully understood. At the transcriptional level, survivin expression has been demonstrated to involve cell-cycle-dependent element/cell cycle gene homology region (CDE/CHR) G1 repressor elements in the BIRC5 (baculoviraliap repeat containing 5) promoter ( Altieriet al.,2003). 
Survivin is expressed in embryonic and foetal tissues, but is undetectable in normal adult tissues(Ambrosiniet al.,1997). However, overexpression of survivin has been reported in almost all human malignancies including bladder cancer, lung cancer, breast cancer, stomach, oesophagus, liver, ovarian cancers and haematological cancers ( Fukuda et al.,2001).

Based on detection of protein by immunohistochemistry and mRNA by polymerase chain reaction techniques, overexpression of survivin has been reported in various human malignancies. Almost all cancers have alternative survivin expression profile compared to normal tissues. Survivin is one of the important genes involved in tumour aggressiveness and therapy resistance. Salzet alshowed that survivin expression induced transcriptional changes in the tissue microenvironment further promoting tumourigenesis in the bladder tissue (Salzet al.,2005). Khan et al reported higher expression of survivin as a critical factor for radioresistance in head and neck squamous cell carcinoma (HNSCC) cell lines(Khan et al.,2010).

Dysregulation of oncoapoptotic genes, growth factors, receptors and their downstream signaling pathway components represent a central driving force in tumour development in different cancer ( Khanet al.,2013).

DNA polymorphisms with more than one variant (allele) having a frequency greater than 1 per cent in a human population have been estimated to occur on the average at one in every 1000 base pairs throughout the human genome (Sherry et al.,1999). The incidences of polymorphism in genomic DNA, their susceptibility to genetic alterations, and the risk of tumor progression in patients with cancer can vary substantially between different racial groups ( Perez et al .,2006 ). Although most polymorphisms are functionally neutral, some affect regulation of gene expression or the function of the coded protein (Costa et al. ,2008).

The survivin gene codifies a multifunctional protein involved in the regulation of the cell cycle and inhibition of the apoptotic pathway, and a polymorphism located in its promoter region is associated with gene regulation. Most of the polymorphic studies are confined in promoter region among which the single nucleotide polymorphism (SNP) at $-31 G / C$ is most studied in all cancers in comparison to other polymorphic sites (Jaiswal et al.,2012).

\section{Role of survivin in cancer therapy}

Due to its role as a cancer gene intersecting multiple cellular networks, survivin has been vigorously used as a cancer drug target ( Reedet al.,2003).Survivin-based vaccination was found to be safe, with no side effects and associated with antigenspecific immunologic responses. Different strategies have been targeted for inhibition of survivinin vivo such as identification of a specifically interacting peptide. This peptide can recognize survivinintracellularly and cause the degradation of the ligand survivin complex. Further, survivin inhibition can be achieved by targeting with nanobased drug delivery devices coupled with biocompatible natural product derived therapeutics(Hirschowitzet al.,2004) . Other strategies under investigation to target survivin include antisense oligonucleotides, siRNA, ribozymes, immunotherapy and small molecular weight molecules (Khan et al.,2012).

\section{P53 AND ITS ANTIBODIES}

In 1982, Crawford et al. first described antibodies against human p53 protein in 9\% of breast cancer patient sera (Crawford et al.,1982). No significant clinical correlation was reported, and, at that time, no information was available concerning mutations of the p53 gene. Caron de Fromentel et al. later found that such antibodies were present in sera of children with a wide variety of cancers. The average frequency was $12 \%$, but this figure increased to $20 \%$ in Burkitt's lymphoma. Those studies, performed in 
the early 1980s, were virtually ignored for more than 10 years because of a lack of interest in p53 during that period. In the early 1990s, it was discovered that the p53 gene is the most common target for molecular alteration in every type of human cancer (Caron de Fromentelet al.,1992). This provoked considerable interest in the study of the p53 protein and its function in normal and transformed cells. It also led to the rediscovery of this humoral response, which had been found in cancer patients. Since 1992, more than 150 papers have been published on p53-Abs. (Levine et al.,1997).

\section{The p53 Protein}

The tumor suppressor p53 is a phosphoprotein barely detectable in the nucleus of normal cells (Benchimol et al.,1982). On cellular stress, particularly that induced by DNA damage, p53 can arrest cell cycle progression, thus, allowing the DNA to be repaired (Kastan et al.,1991) or it can lead to apoptosis (Yonish-Rouachet al.,1991).

These functions are achieved, in part, by the transactivational properties of $\mathrm{p} 53$, which activate a series of genes involved in cell cycle regulation. In cancer cells that bear a mutant $\mathrm{p} 53$, this protein is no longer able to control cell proliferation, which results in inefficient DNA repair and the emergence of genetically unstable cells. The most common changes of p53 in human cancers are point missense mutations within the coding sequences of the gene. Such mutations are found in all of the major histogenetic groups, including cancers of the colon, stomach, breast, lung, brain, and esophagus (Caron de Fromentelet al.,1992) .

It is estimated that p53 mutations is the most frequent genetic event in human cancers and accounts for more than $50 \%$ of cases. More than $90 \%$ of the point mutations reported thus far are clustered between exons 4 and 10 and are localized in the DNA binding domain of the p53 protein ( Be'roud et al.,1998).

\section{P53 antibodies}

P53-Abs were discovered 20 years ago during the course of tumor-associated antigens screening. The discovery of p53 mutation and accumulation of p53 in human tumors shed new light on the p53 humoral response. It is demonstrated that p53-Abs are found predominantly in human cancer patients with a specificity of $96 \%$. Such antibodies are predominantly associated with p53 gene missense mutations and p53 accumulation in the tumor, but the sensitivity of such detection is only $30 \%$. It has been demonstrated that this immune response is due to a self-immunization process linked to the strong immunogenicity of the p53 protein. The clinical value of these antibodies remains subject to debate, but consistent results have been observed in breast, colon, oral, and gastric cancers, in which they have been associated with highgrade tumors and poor survival. The finding of p53-Abs in the sera of individuals who are at high risk of cancer, such as exposed workers or heavy smokers, indicates that they have promising potential in the early detection of cancer. (Angelopoulou et al.,1994).

\section{The Specificity of p53 Antibodies in Cancer Patients}

The initial work on p53-Abs used either immunoprecipitation or Western blot as the detection method (Caron de Fromentel et al.,1987). Later, several ELISAs were developed to handle large numbers of specimens, and some of them are now commercially available (Lubin et al,1995).

One of the most important parameters seems to be the antigen used for these assays. It has been shown that p53-Ab recognized immunodominant epitopes localized in the $\mathrm{NH} 2$ and $\mathrm{COOH}$ termini of the protein. It is thus essential to use the entire p53 protein as antigen. Several attempts to develop an ELISA with synthetic 
peptides corresponding to these immunodominant epitopes have been unsuccessful, because they lead to a high level of false-negative results (Vennegoor et al.,1997).

\section{P53 Antibodies: Future Directions}

There exist several situations in which p53-Abs could have clinical utility. The first is in the monitoring of sera during therapy. Only prospective studies on various types of cancer in which relapses occur several months or years after treatment will enable us to validate this assay. The use of standardized assays that have been validated for quantitative analysis should help in such studies. The second situation concerns p53Abs in high-risk individuals. One of the challenges of the next millennium is the early detection of tumors using highly sensitive assays with gene probes specific for tumor genetic alterations (Mao et al.,1997).

Conclusion: So for finding a useful marker to identify genetic susceptibility, polymorphism of survivin gene might be of help since survivin acts as an inhibitor of apoptosis which is essential for eliminating mutated or transformed cells from the body, thus it may be possible that subjects with a lower production genotype for survivin have increased apoptotic capacity to eliminate cells with DNA damage and have decreasedsusceptibility to breast cancer. This polymorphism may depress survivin transcription by modifying the binding motif of the $\mathrm{CDE} / \mathrm{CHR}$ repressor.The presence of p53 Abs is probably triggered by the accumulation of tumor p53 protein, and it could be a useful marker to complement routine prognostic factors in breast cancer patients.

\section{Referrences}

1. Altieri DC.,(2003):Survivin, versatile modulation of cell division and apoptosis in cancer. Oncogene ; 22 : 8581-9.

2. Ambrosini G, Adida $\mathrm{C}$ and Altieri DC.,(1997): A novel anti-apoptosis gene, survivin, expressed in cancer and lymphoma. Nat Mad ; 3 : 917-21.

3. Angelopoulou, K., Diamandis, E. P., Sutherland, D. J. A., Kellen, J. A., and Bunting, P. S.,(1994): Prevalence of serum antibodies against the p53 tumor suppressor gene protein in various cancers. Int. J. Cancer, 58: 480-487.

4. Be'roud, C., and Soussi, T.(1998): p53 gene mutation: software and database. Nucleic Acids Res., 26: 200204.

5. Caron de Fromentel, C., May-Levin, F., Mouriesse, H., Lemerle, J., Chandrasekaran, K., and May, P. (1987): Presence of circulating antibodies against cellular protein p53 in a notable proportion of children with B-cell lymphoma. Int. J. Cancer, 39: 185-189.
6. Fukuda $S$ and Pelus LM.,(2001): Regulation of the inhibitor-of-apoptosis family member survivin in normal cord blood and bone marrow CD34(+) cells by hematopoietic growth factors: implication of survivin expression in normal hematopoiesis. Blood ;98:2091 -100 .

7. Hoffmann, L.S., Etzrodt, J., Willkomm, L., Sanyal, A., Scheja, L., Fischer, A.W.C., Stasch, J.-P., Bloch, W., Friebe, A., Heeren, J., and Pfeifer, A. (2015).Stimulation of soluble guanylylcyclase protects against obesity by recruiting brown adipose tissue. Nat. Commun. 6, 7235.

8. Khan $Z$ and Bisen PS.,(2013): Oncoapoptotic signaling and deregulated target genes in cancers: special reference to oral cancer. BiochimBiophysActa 2013; 1836 : 123-45. 48.

9. Lubin, R., Schlichtholz, B., Teillaud, J. L., Garay, E., Bussel, A., Wild, C., and Soussi, T. (1995): p53 antibodies in patients with various types of cancer: assay, identification and 
SOHAG MEDICAL JOURNAL

Vol. 23 No.2Apr 2019

characterization. Clin. Cancer Res., 1: 1463-1469.

10.Reed JC.(2003):Apoptosis-targeted therapies for cancer. Cancer Cell 2003; 3 : 17-22.

11.Siegel $R$, Naishadham D, Jemal A. Cancer statistics, 2013. CA: a cancer journal for clinicians. Jan 2013;63(1):11-30.

12.Monticciolo DL, Newell MS, Moy L, Niell B, Monsees B, Sickles EA.J Am CollRadiol.,(2018): Breast Cancer Screening in Women at Higher-ThanAverage Risk: Recommendations From the ACR. Mar;15(3 Pt A):408-414. doi: 10.1016/j.jacr.2017.11.034.
Survivin -31G/C promoter polymorphism

13.Mao, L., Lee, J. S., Kurie, J. M., Fan, Y. H., Lippman, S. M., Lee, J. J., Ro, J. Y., Broxson, A., Yu, R., Morice, R. C., Kemp, B. L., Khuri, F. R., Walsh, G. L., Hittelman, W. N., and Hong, W. K. (1997): Clonal genetic alterations in the lungs of current and former smokers. J. Natl. Cancer Inst., 89: 857-862.

14.Yonish-Rouach, E., Resnitzky, D., Lotem, J., Sachs, L., Kimchi, A., and Oren, M. (1991): Wild-type p53 induces apoptosis of myeloid leukaemic cells that is inhibited by interleukin-6. Nature (Lond.), 352: 345-347. 